\title{
Design and Simulation of a Plasmonic Refractive Index Nano-Sensor Based on Tunable Fano Resonance
}

\section{Babak Moeinimaleki}

Amirkabir University of Technology Department of Electrical Engineering

Hassan Kaatuzian ( $\nabla$ hsnkato@aut.ac.ir)

Amirkabir University of Technology Department of Electrical Engineering https://orcid.org/0000-00025381-6455

Abdolber Mallah Livani

University of Science and Technology of Mazandaran

\section{Research Article}

Keywords: plasmonic sensor, figure of merit, Fano resonance, Metal-Insulator-Metal (MIM) waveguide

Posted Date: February 1st, 2022

DOI: https://doi.org/10.21203/rs.3.rs-1274109/v1

License: (a) (1) This work is licensed under a Creative Commons Attribution 4.0 International License. Read Full License 


\title{
Design and Simulation of a Plasmonic Refractive Index Nano- Sensor based on Tunable Fano Resonance
}

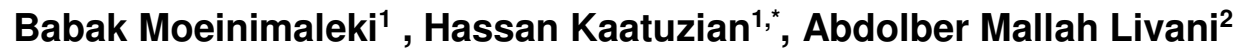 \\ ${ }^{1}$ Photonics Research Lab, Electrical Engineering Department, Amirkabir University of Technology, Tehran, Iran. \\ ${ }^{2}$ Electrical and Computer Engineering Department, University of Science and Technology of Mazandaran, Behshahr, Iran. \\ *Corresponding author: Hassan Kaatuzian \\ hsnkato@aut.ac.ir \\ first author: Babak Moeinimaleki \\ b.moeinimaleki@aut.ac.ir \\ Abdolber Mallah Livani \\ mallah_a@mazust.ac.ir
}

\begin{abstract}
In this paper, a nano-scaled surface plasmon-polariton (SPP) refractive index sensor based on metal-insulator-metal (MIM) waveguide is proposed and evaluated numerically by finite-difference time-domain (FDTD) method. Polygonal cavities, that are coupled to a disk resonator cavity, are attached to this sensor's MIM bus waveguide. Fano resonance and its characteristics are utilized to improve the essential parameters of the sensor, such as full width at half maximum (FWHM), quality factor, and sensitivity. Figure of merit (FOM) of the sensor can be increased by tuning its geometrical parameters. In optimized conditions, which are concluded in this work, the sensor shows a FWHM of $1.15 \mathrm{~nm}$, a quality factor of 604.1, a sensitivity of $687.9 \mathrm{~nm} / \mathrm{RIU}$ and a FOM of $598.1 \mathrm{RIU}^{-1}$ in the visible light region. A sensor with such a high resolution can be used in highly integrated photonic circuits and biosensors. The effects of incorporating metal patches inside the disk cavity are also discussed in this paper.
\end{abstract}

Keywords plasmonic sensor; figure of merit; Fano resonance; Metal-Insulator-Metal (MIM) waveguide.

\section{Introduction}

Surface Plasmon Polaritons (SPPs) are electromagnetic waves coupled to the oscillations of the free electrons at the surface of metal (Kaatuzian, Taheri 2015). SPPs propagate along with the interface of the metal-insulator (Maier 2007). Plasmonic sensors can be made in nano-scaled dimensions (Ozbay 2006). This is because SPPs can overcome diffraction limit (Barnes et al. 2003), and therefore they are appropriate for highly integrated photonic and plasmonic circuit applications. Plasmonic devices such as optical switches (Khani et al. 2021; Wang et al. 2012; Karimi et al. 2021), splitters (He et al. 2010; Yu et al. 2017), demultiplexers (Wen et al. 2012; Hajshahvaladi et al. 2019), filters (Wu et al. 2016; Shafagh et al. 2020), and sensors (Wang et al. 2019) have been investigated frequently. Lately, plasmonic sensors have drawn attention due to their smaller size and also analogous operational parameters compared to other optical sensors (Zhang et al. 2018). Plasmonic sensors have been categorized into refractive index sensors (Butt et al. 2019; Yu et al. 2019; Chen, Yu 2014), temperature sensors (Wu et al. 2015), phase sensors (Ouyang et al. 2017), gas sensors (Elsayed et al. 2017; Butt et al. 2021), and biosensors (Setareh, Kaatuzian 2021).

The Fano resonance phenomenon has recently found its way to the plasmonic devices (Wang et al. 2019; Yu et al. 2019; Fang et al. 2019; Deng et al. 2018; Chen et al. 2019). The distinctive asymmetric shape and having high 
sensitivity to the surrounding environment and geometrical changes are characteristics that are mentioned for the Fano resonances (Luk'yanchuk et al. 2010; Lu et al. 2012).

Several studies have demonstrated structures such as the MIM waveguide side coupled with a ring or disk resonator, which could be applied as a refractive index sensor (Wang et al. 2019; Zhang et al. 2018; Yu et al. 2019; Zhang et al. 2020). In our previous paper (Moeinimaleki et al. 2021) design of a nano-scaled plasmonic waveguide temperature sensor coupled to a rectangular cavity (filled with ethanol) equipped with polygonal metal patches positioned along the cavity has been proposed Which showed the ability of concentrating the fields at its patches' sharp edges in the cavity to enhance the sensing mechanism and resulted in $0.97 \mathrm{~nm} /{ }^{\circ} \mathrm{C}$ sensitivity.

In this paper, a Metal Insulator Metal (MIM) waveguide which supports SPP modes (Lu et al. 2013) is applied for the proposed sensor. Two pentagon-shaped cavities are attached to the top, and one rectangular cavity is attached to the bottom of the MIM waveguide. These cavities are coupled to a disk resonator which is located above the two pentagon cavities. At this work for brevity, the system of MIM waveguide side-coupled to a disk resonator is called the Primitive System. Also, the MIM waveguide attached to the two pentagon cavities and one bottom rectangular cavity is called the Waveguide System. Additionally, the Total System is defined as the waveguide system coupled to the disk resonator.

FWHM and subsequently FOM are two parameters whose importance is ignored in favor of sensitivity in the previous works (Butt et al. 2019, 2021; Yan et al. 2017; Butt et al. 2018; Zhang et al. 2016; Akhavan et al. 2018). A good sensor should have both high sensitivity and high FOM simultaneously. Since the Fano resonance is highly sensitive to geometrical changes (Luk'yanchuk et al. 2010), optimization of the sensor's operational parameters such as sensitivity, FWHM, and FOM has been performed by adjusting its geometrical variables. This work proposes a plasmonic sensor with a sensitivity of $687.9 \mathrm{~nm} / \mathrm{RIU}$ and a FOM of $598.1 \mathrm{RIU}^{-1}$ at the visible light region. Simulations are performed by the two-dimensional finite-difference time-domain (2D FDTD) method, and the Perfectly Matched Layer (PML) boundary conditions are applied to probe the sensing properties.

This paper is organized as follows. In section 2, the proposed sensor structure with its geometrical parameters and also theoretical analysis will be explained in detail. In section 3, induced Fano resonances of the total system and their operational parameters like sensitivity, FWHM, and FOM will be evaluated. Also, the special case of the total system equipped with metal patches inside the disk resonator will be presented. Additionally, the stability of the proposed sensor will be evaluated. And the final section is for conclusions.

\section{Proposed structure and theoretical analysis}

As depicted in Fig. 1a, a MIM waveguide is attached to three polygonal cavities which are coupled to a disk resonator. The blue and white areas in this figure represent silver and the substance that is supposed to be sensed, respectively. A silver film on the top of the silicon substrate has been selected for this sensor, which is a conventional setting in devices that are based on MIM waveguide (Zhang et al. 2018; Butt et al. 2021; Zafar, Salim 2015). The geometrical parameters of the sensor structure are collected in Table 1. The experimental permittivity data of Johnson and Christy are applied for modeling the silver material (Ag) in simulations (Johnson, Christy 1972). The simulations in this paper were 2D, and the Ag film thickness of the sensor had no impact on the results. However, in fabrication, the minimum height of $50 \mathrm{~nm}$ should be considered for the silver layer (Zhang et al. 2018). In order to fabricate the MIM waveguide and its associated cavities and disk resonator, an etching method has to be applied on silver's surface (Kazanskiy et al. 2020). Such fabrication demands a method with high etching precision due to the small features of the proposed structure (Table 1). For the fabrication of the proposed structure, the waveguide and cavities can be etched on the surface of silver which is previously deposited on a silicon substrate (Zhang et al. 2018). This structure can be 
fabricated by the vertical bombardment of heavy ions on the top layer of silver by the Focused Ion Beam (FIB) method (Gierak et al. 2007).
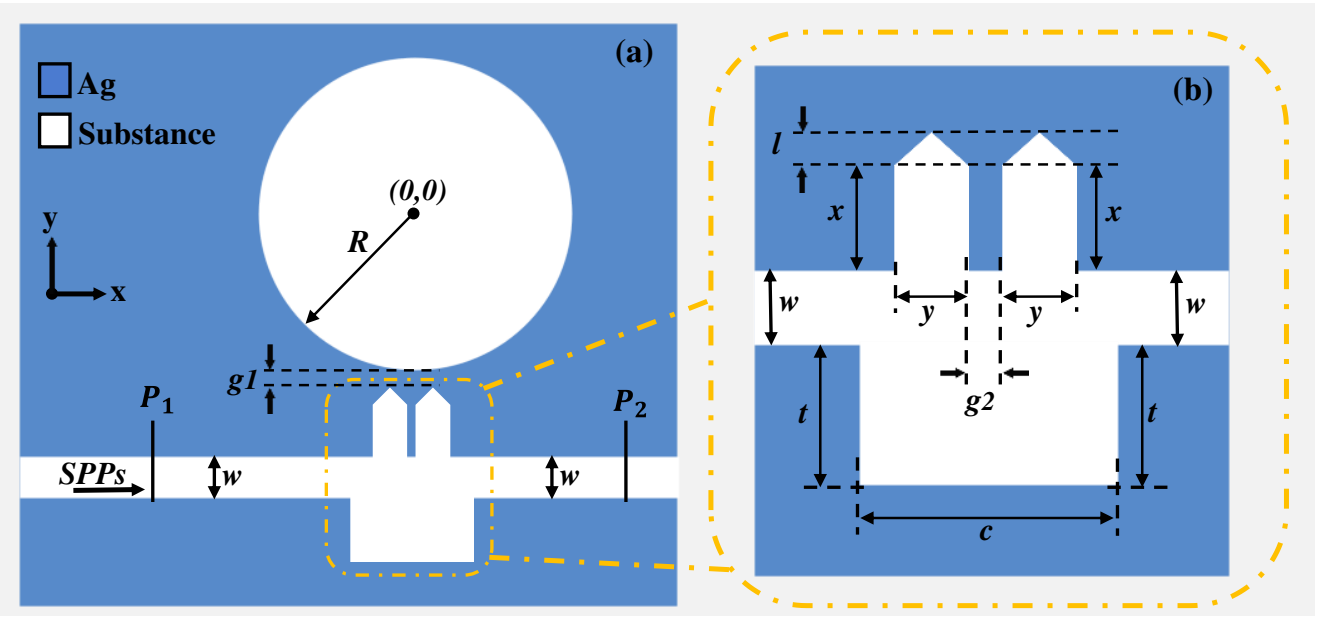

Fig. $12 \mathrm{D}$ cross-sectional view of the proposed nano-scaled plasmonic refractive index sensor: a The total system, $\mathbf{b}$ The waveguide system.

Table 1 The geometrical parameters of the sensor structure.

\begin{tabular}{clcc}
\hline Symbol & & Value & Unit \\
\hline$R$ & The radius of the disk & 400 & $\mathrm{~nm}$ \\
$w$ & Width of the MIM waveguide & 100 & $\mathrm{~nm}$ \\
$c$ & Width of the bottom rectangular cavity & 230 & $\mathrm{~nm}$ \\
$t$ & Height of the bottom rectangular cavity & 180 & $\mathrm{~nm}$ \\
$g_{1}$ & The gap between the disk and the waveguide system & 10 & $\mathrm{~nm}$ \\
$g_{2}$ & The gap between the two pentagon cavities & 10 & $\mathrm{~nm}$ \\
$x$ & Height of rectangular section of the pentagon cavities & 50 & $\mathrm{~nm}$ \\
$y$ & Width of rectangular section of the pentagon cavities & 25 & $\mathrm{~nm}$ \\
$l$ & Height of triangular section of the pentagon cavities & $\mathrm{nm}$ \\
\hline
\end{tabular}

The theoretical dispersion relation for the MIM waveguide is as follows (Maier 2007).

$$
\begin{gathered}
\frac{\varepsilon_{i} k_{m}}{\varepsilon_{m} k_{i}}=\frac{1-e^{k_{i} w}}{1+e^{k_{i} w}} \\
k_{i}=\sqrt{\beta_{s p p}^{2}-\varepsilon_{i} k_{0}^{2}}, k_{m}=\sqrt{\beta_{s p p}^{2}-\varepsilon_{m} k_{0}^{2}} \\
\beta_{s p p}=n_{e f f} k_{0}=n_{e f f} \frac{2 \pi}{\lambda}
\end{gathered}
$$

Where $w$ is the width of the MIM waveguide, $\lambda$ is the free space wavelength of the incident light, $\varepsilon_{i}$ and $\varepsilon_{m}$ are the permittivities of the dielectric and metal, respectively, $k_{i}$ and $k_{m}$ are transverse propagating constants in dielectric and metal, respectively, $\beta_{s p p}$ and $n_{e f f}$ are propagating constant and effective refractive index of SPPs, respectively, and $k_{0}=\frac{2 \pi}{\lambda}$ is the wave vector of the incident light in free space. 
The resonance condition for the primitive structure of a MIM waveguide side-coupled to a single disk is described by equation (4) (Zhan et al. 2014). However, in more sophisticated cases where structures contain polygonal cavities or metal patches, numerical methods are needed for finding the resonance condition.

$$
k_{i, d} \frac{H_{n}^{(1) \prime}\left(k_{m, d} r\right)}{H_{n}^{(1)}\left(k_{m, d} r\right)}=k_{m, d} \frac{J_{n}{ }^{\prime}\left(k_{i, d} r\right)}{J_{n}\left(k_{i, d} r\right)}, \quad n=1,2,3, \ldots
$$

Where $\mathrm{r}$ is the radius of the disk, $k_{i, d}$ and $k_{m, d}$ represent the wave vectors of the propagating modes in the dielectric and metal which are located inside the disk, respectively. $H_{n}^{(1)}$ and $H_{n}^{(1)^{\prime}}$ are the first kind Hankle's function with the order of $n$ and its first derivative, respectively. $J_{n}$ and $J_{n}{ }^{\prime}$ are the Bessel's function of the first kind with the order of $n$ and its first derivative, respectively, and $n$ represents the mode number.

\section{Results and discussions}

\subsection{Transmission spectra of the waveguide system}

In Figs. 2a, b, and c, the transmission spectra of the proposed waveguide system is compared with its modifications. The corresponding structure of each transmission is depicted in the insets of these figures. FDTD method is used for extracting the results that are provided in all subsections of section 3. The geometrical parameters' values that are considered in the simulations related to the results that are provided in section 3 are the same as the values mentioned in Table 1. however, when effects of specific geometrical parameter on transmission spectra of the proposed device are provided by its several values, those values are mentioned in the inset of their corresponding figure.

In Fig. 2a, the solid blue line, the red dashed line, and the black dotted line indicate the transmission spectra of the waveguide system, the waveguide system without the rectangular cavity, and the waveguide system without the two pentagon cavities, respectively. Fig. $2 b$ evaluates the impact of different numbers of pentagon cavities of the waveguide system on its transmission spectra. The solid blue line, the red dashed line, and the black dotted line indicate the transmission spectra of the waveguide system (with two pentagon cavities), the waveguide system with one pentagon cavity, and the waveguide system with three pentagon cavities, respectively. Fig. $2 \mathrm{c}$ is dedicated to evaluating the effects of bottom cavities of the same area but different shapes of the waveguide system on its transmission spectra. The solid blue line and the red dashed line indicate the transmission spectra of the waveguide system (with the bottom rectangular cavity) and the waveguide system with a bottom round cavity (a semicircular cavity with a radius of $162 \mathrm{~nm}$ ), respectively.

Figs. $2 \mathrm{~d}$ and e represent the normalized real parts of the $\mathrm{z}$ component of the magnetic fields $\left(\mathrm{H}_{\mathrm{z}}\right)$ at $\lambda=912.2 \mathrm{~nm}$ and $\lambda=932.4 \mathrm{~nm}$ for transmission spectra of the waveguide system, respectively. In the case in Fig. 2e, that transmission spectra have a local minimum (almost zero) at $\lambda=932.4 \mathrm{~nm}$, it can be inferred that the phase shift of SPPs due to these three cavities in the waveguide system causes destructive interference of them. Subsequently, transmission intensity at this mentioned wavelength is suppressed. This is in spite of the case in Fig. 2d which transmission spectra of the waveguide system have a local maximum at $\lambda=912.2 \mathrm{~nm}$. 

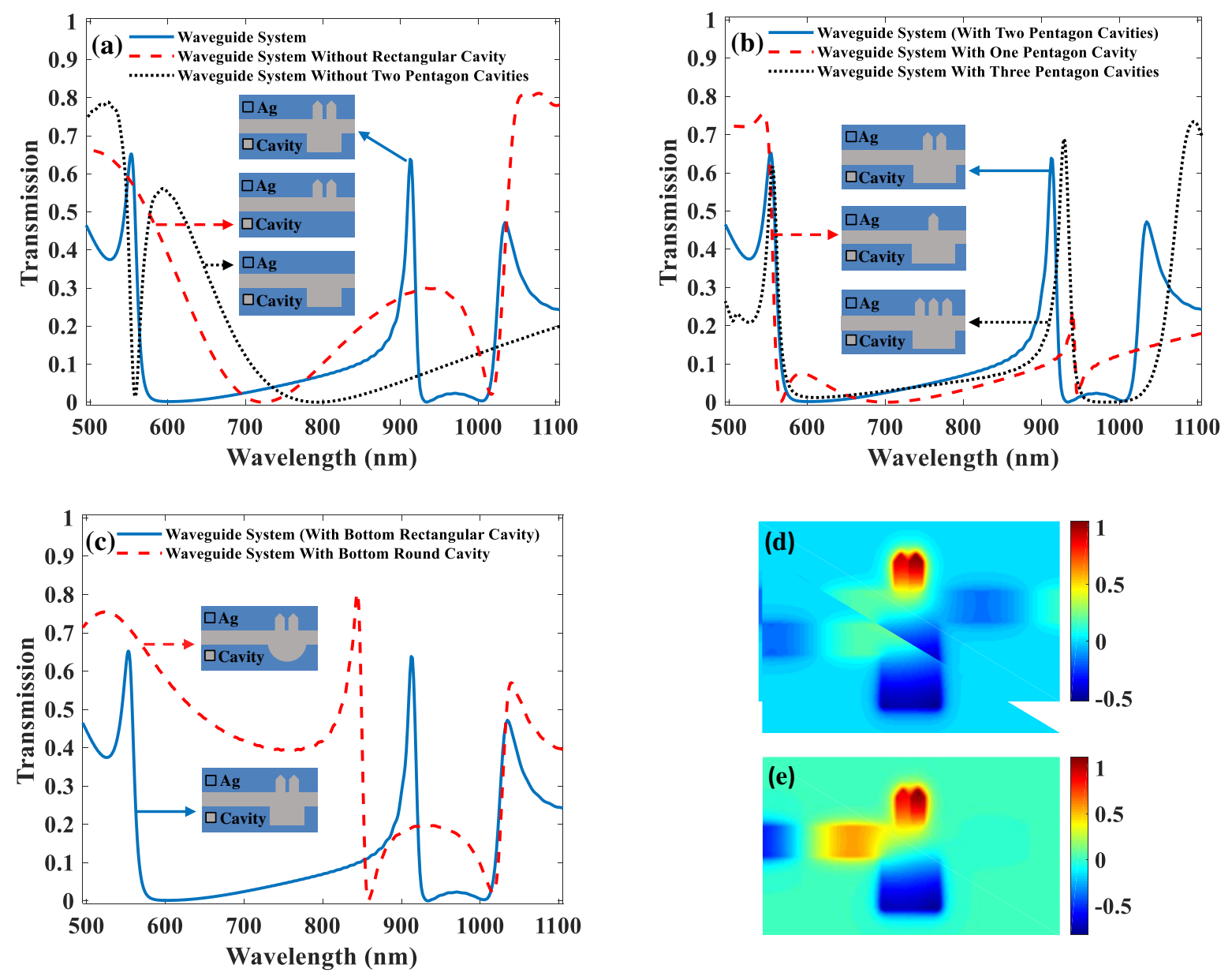

Fig. 2 Based on Table 1 values of the parameters: The transmission spectra of the waveguide system and a two cases of the waveguide system without one bottom rectangular or two pentagon cavities, $\mathbf{b}$ two cases of the waveguide system with one or three pentagon cavities, $\mathbf{c}$ the waveguide system with a bottom round cavity (a semicircular cavity with a radius of $162 \mathrm{~nm}$ ). The insets in $\mathbf{a}, \mathbf{b}$, and $\mathbf{c}$ show the corresponding structure of each depicted transmission. $\mathbf{d}$ and $\mathbf{e}$ show 2D distribution of the normalized real parts of the $H_{z}$ at $\lambda=912.2 \mathrm{~nm}$ and $\lambda=$ $932.4 \mathrm{~nm}$ for the transmission spectra of the waveguide system, respectively.

\subsection{Transmission spectra of the total system and its induced Fano resonances}

It is understood that Fano resonance arises from the constructive and destructive interferences of a narrow discrete resonance with a broad spectral line or continuum (Luk'yanchuk et al. 2010). As it is shown in the transmission spectra of the primitive system in Fig. 3a, multiple narrow discrete resonances can be induced by the disk resonator. Since the disk cavity and waveguide system are coupled together, for inducing Fano resonances at Transmission Spectra of the total system, it is needed for the waveguide system to be designed in a way to have an interested broad spectral line in the arbitrary wavelength interval. In Figs. 2a, b, and c, the transmission spectra of the waveguide system (solid blue line) is compared with the other five modifications of it. The waveguide system is preferred to other options because it is more compatible with the feature of having a broad spectral line in the visible light region where the disk cavity provides two discrete resonances.

By incorporating the disk cavity on top of the waveguide system (Fig. 1a), the narrow discrete resonances induced by the disk cavity and the broad spectral line induced by the waveguide system interfere together. Thus, according to the Fano resonance theory and by obtaining the numerical results, some Fano resonances at the transmission spectra of the total system can be observed, as depicted in Fig. 3a. 

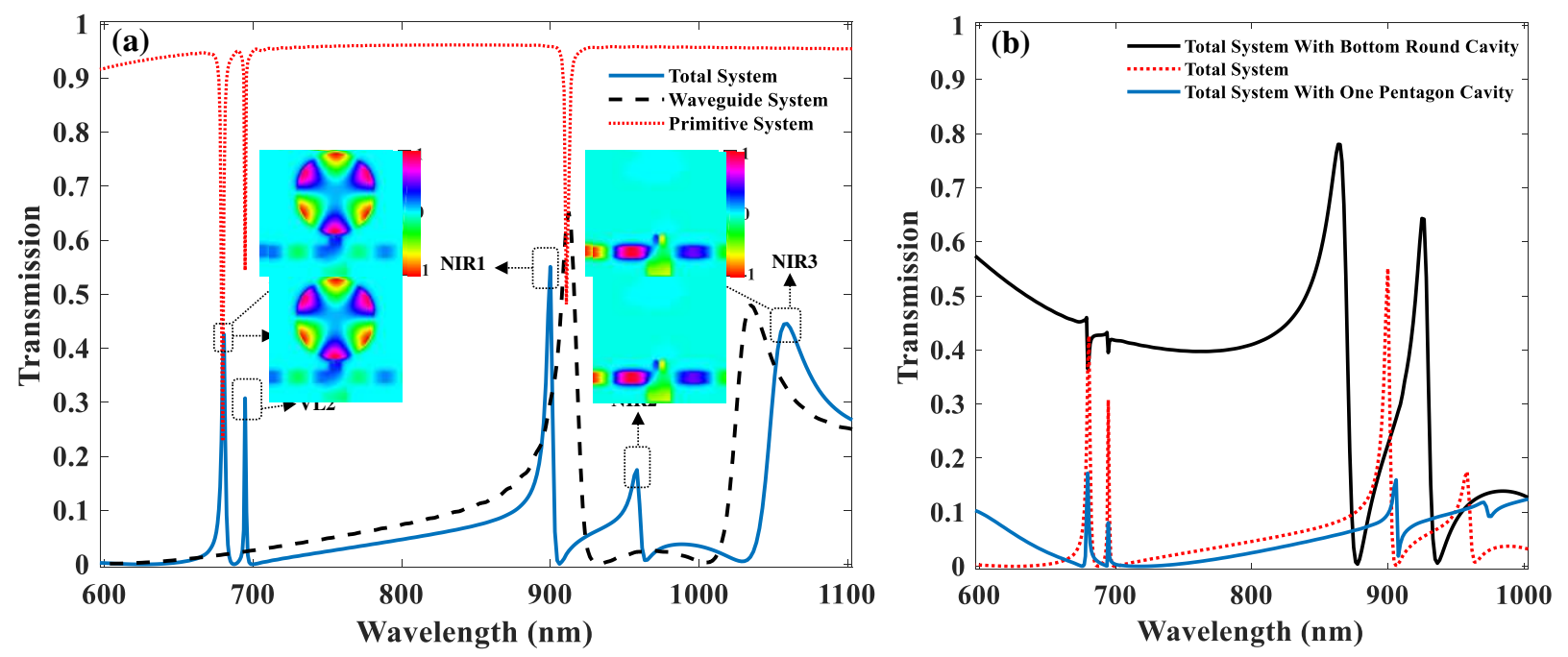

Fig. 3 Based on Table 1 values of the parameters: a The transmission spectra of the total system, the waveguide system, and the primitive system. In a, the insets show the 2D distribution of the real parts of $H_{z}$ for the transmission spectra of the total system at $\lambda=680.2 \mathrm{~nm}$ and $\lambda=1058.5 \mathrm{~nm}$. $\mathbf{b}$ The induced Fano resonances at the transmission spectra of the total system, the total system with a bottom round cavity (a semicircular cavity with a radius of $162 \mathrm{~nm}$ ), and the total system with only one pentagon cavity.

In Fig. 3a, the red dotted line corresponds to the transmission spectra of the MIM waveguide side-coupled to a disk resonator (the primitive system) with a gap of $30 \mathrm{~nm}$ between them, the black dashed line corresponds to the transmission spectra of the waveguide system, and the blue solid line corresponds to the transmission spectra of the total system. As it is obvious from Fig. 3a, four Fano resonances, that have occurred due to the interference of the narrow discrete resonances induced by the disk cavity with the broad spectral line induced by the waveguide system, can be distinguished, and they are named VL1, VL2, NIR1, and NIR2. However, resonance that is named NIR3 in Fig. $3 \mathrm{a}$ is induced only by the waveguide system itself. The presence of VL or NIR in the name of a resonance denotes that it belongs to visible light or near infra red regions, respectively. Insets of Fig. 3a show the normalized real parts of the $\mathrm{H}_{\mathrm{z}}$ for transmission spectra of the total system at its two resonance wavelengths of VL1 and NIR3. At the inset that is related to the resonance of VL1 $(\lambda=680.2 \mathrm{~nm})$, it can be observed that SPPs are engaged with both the waveguide system and the disk cavity. On the other hand, the inset that is depicted at the resonance wavelength of NIR3 $(\lambda=1058.5 \mathrm{~nm})$ shows that the SPPs are only engaged with the waveguide system. These two results are consistent with the Fano resonance theory.

Fig. 5b compares the induced Fano resonances of the total system and two of its modifications. The red dotted line, the black solid line, and the blue solid line indicate transmission spectra of systems of the disk cavity coupled with the waveguide system, the waveguide system with a bottom round cavity (a semicircular cavity with a radius of $162 \mathrm{~nm}$ ), and the waveguide system with one top pentagon cavity, respectively.

\subsection{Adjusting the geometrical parameters of the total system}

Transmission spectra of the total system varies by changing its geometrical parameters, and thus it affects the performance of the sensor. Figs. $4 \mathrm{a}, \mathrm{b}$, and $\mathrm{c}$ depict the impacts of the variations of the three geometrical parameters on the transmission spectra of the total system. Changing of $x$ or $g_{2}$ (belonging to the waveguide system) shifts the wavelength of the resonance of the waveguide system, which is located between the two peaks of NIR1 and NIR2 (Fig. 3a). Interference of the shifted resonance of the waveguide system with the fixed resonance of the disk cavity (According to equation $4, R$ or the radius of the disk cavity is the only geometrical parameter that determines the resonance wavelength. The $R$ is fixed at $400 \mathrm{~nm}$ for all cases of Fig. 4) leads to shifted Fano resonances of NIR1 and NIR2. These shifts can be observed for the NIR1 resonance in Figs. 4b and c. But, the NIR1 resonance barely shifts by the variation of $g_{1}$ as it is clear in Figure 5.a. This is because the parameter of $g_{1}$ does not belong to the waveguide 
system and thus its variation does not affect the resonance wavelengths at transmission spectra of the waveguide system. Moreover, since the resonance wavelengths of the disk cavity are fixed (by fixing $\mathrm{R}$ at $400 \mathrm{~nm}$ ), and the transmission spectra of the waveguide system is linear and with no resonance in the visible light region (Fig. 3a), the interaction of the SPPs' modes between the waveguide system and the disk cavity leads to Fano resonances with fixed wavelength at each mentioned step of $g_{1}, g_{2}$ and $x$ in Figs. 4a, b and c for VL1 and VL2.
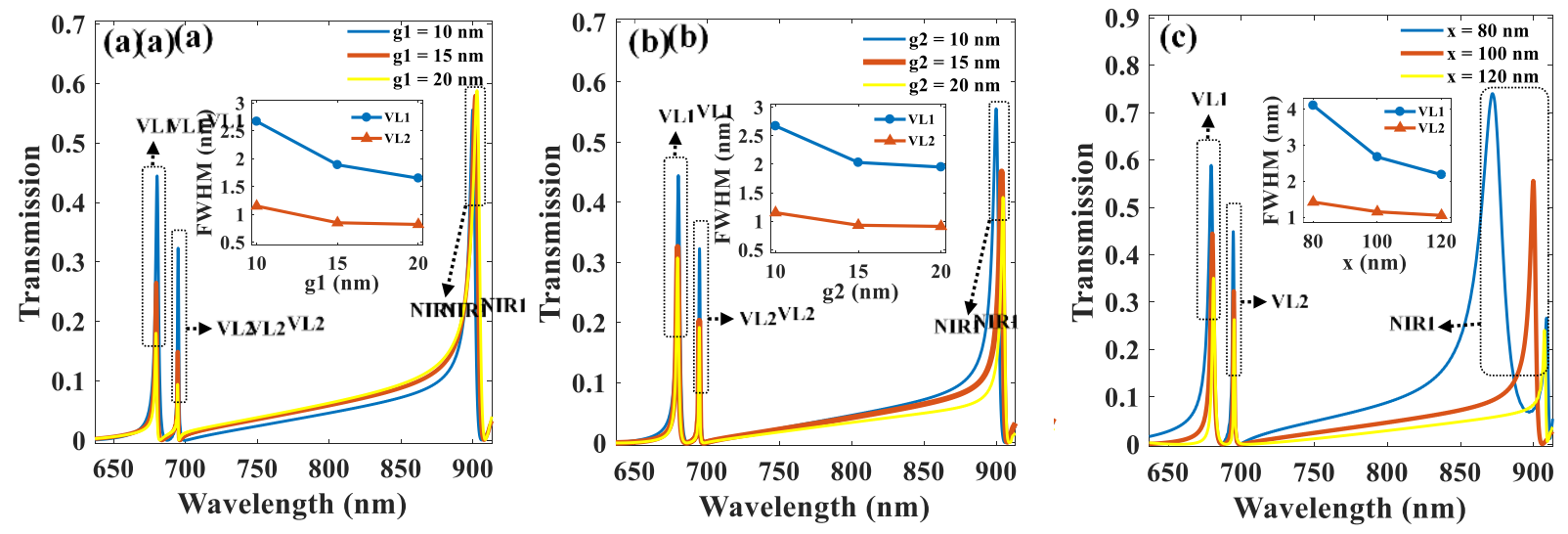

Fig. 4 The transmission spectra of the total system at three different values for $\mathbf{a} g_{1}, \mathbf{b} g_{2}$, and $\mathbf{c} x$. The insets show the FWHM of the two resonances at VL1 and VL2. The fixed values for $g_{1}, g_{2}$ and $x$ and other geometrical parameters are based on Table 1 values of the parameters.

The insets of Fig. 4 show the FWHMs of the two resonances in the visible light region (VL1 and VL2) in each step. The relative stability of the resonance wavelengths and the FWHMs of these resonances makes this structure a reliable option for fabrication. It is obvious from Fig. 4 that for the resonances of VL1 and VL2, increasing the values of $x$, $g_{1}$, and $g_{2}$, will reduce the FWHMs of them. Subsequently, their Q-factors which is defined as $\lambda /$ FWHM (Zhang et al. 2018), will increase ( $\lambda$ is almost fixed for VL1 and VL2 at each mentioned step of $g_{1}, g_{2}$ and $x$ ). But this happens at the cost of decrease in their transmission intensity. By accepting that there is a trade-off between the Q-factor and the transmission intensity, the optimum values of $x=100 \mathrm{~nm}$ and $g_{1}=g_{2}=10 \mathrm{~nm}$ are chosen which lead to the FWHM of $2.67 \mathrm{~nm}$ and the Q-factor of 254.7 for VL1, and the FWHM of $1.15 \mathrm{~nm}$ and the Q-factor of 604.1 for VL2.

\subsection{Tunable characteristic of the Fano resonances in the proposed structure}

Another parameter of the sensor that is evaluated in this work is the wavelength difference of the two resonance peaks at VL1 and VL2, which can be defined as free spectral range (FSR) between the two mentioned resonances. This parameter is adjusted by adding metal patches inside the disk cavity. In the case of Fig. 5e, where two triangular metal patches are added inside the disk cavity, at the resonance of VL2, the electric field is mainly concentrated at the two base edges of the triangular metals while its magnitude at the vertex edges of them is almost zero. Therefore, for adjusting of resonance of VL2, two parameters of the base of metal triangles (parameter of $b$ ) and their position with respect to the center of the disk (parameter of $d$ ) are chosen. As mentioned, the magnitude of electric field in vertex points of two metal triangles are almost zero (Fig. 5e), which means that the parameter of the height of metal triangles (h) has less control on the adjustment of the resonance of VL2 since there is no interaction of light and matter in those areas. Therefore, this parameter is ignored to be evaluated.

As it is depicted in Fig. 5e, the electric field is inclined to concentrate at the sharp section of metal patches, and it could be inferred that the polygonal metal shapes other than triangles, which also include sharp edges, could be applied to adjustment of VL2. However, it is important to be mentioned that in that case, since the sides of the shapes increase, the number of parameters to be evaluated increases. Moreover, as depicted in Fig. 5a, the parameter of the base of two triangles alone controls the FSR between VL1 and VL2, and therefore triangular metal patches are chosen for this work. However, with techniques such as varying the radius of the disk or ring cavity, the FSR between all resonances can be adjusted (Ou et al. 2021), which would cause the transmission spectra and subsequently operational parameters 
of the sensor to change remarkably, but to our knowledge, this is the first time that position (and subsequently FSR) of a specific resonance is adjusted while other resonances are relatively fixed which is done by merely incorporating the metal patches inside the disk cavity and without manipulating the main geometrical parameters of the sensor (such as radius of the disk or ring resonator).
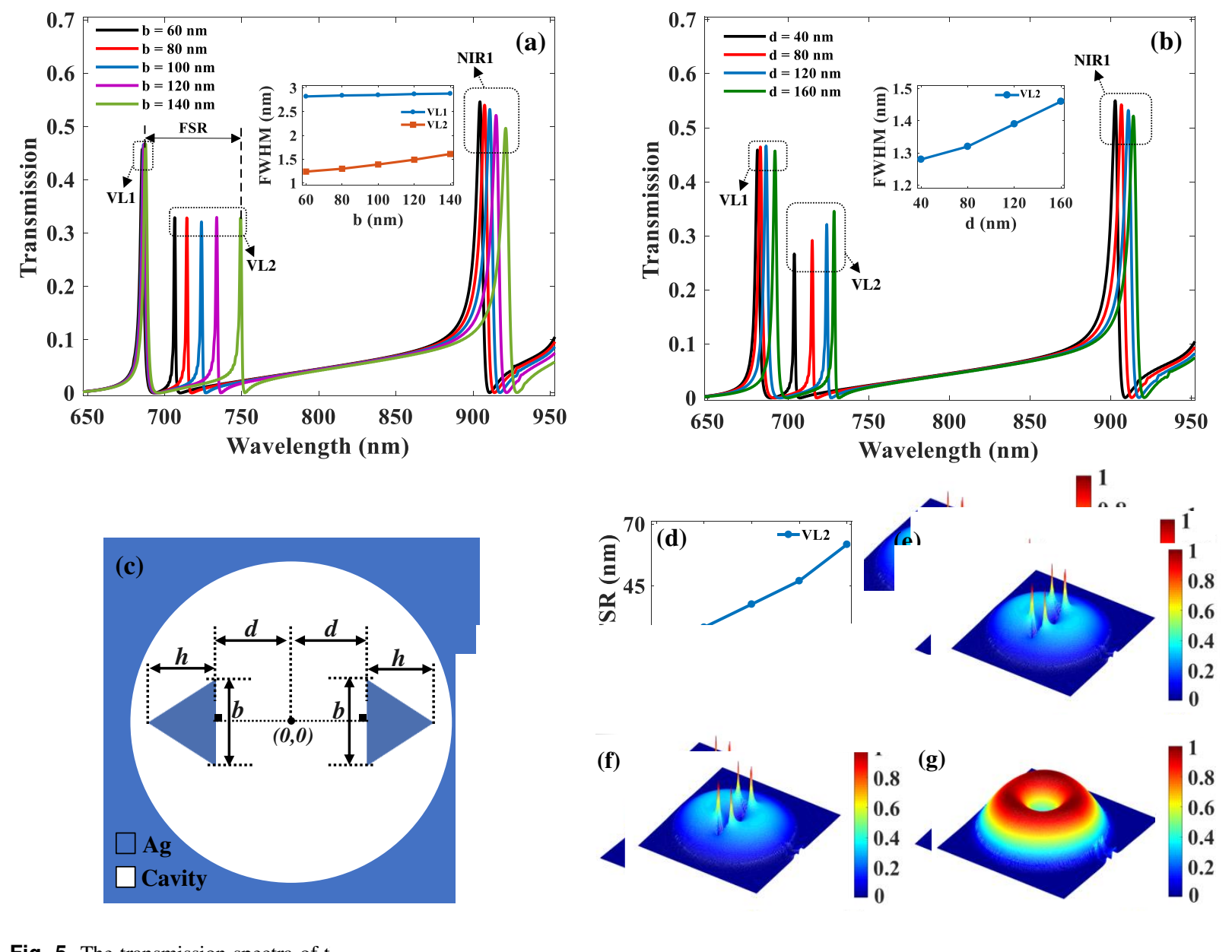

Fig. 5 The transmission spectra of the total system with two triangular silver patches inside the disk cavity: a For five distinct values of $b$ parameter, $\mathbf{b}$ For four distinct values of $d$ parameter. The inset of $\mathbf{a}$ shows FWHMs of the resonances at VL1 and VL2 as a function of the parameter of $b$. The inset of $\mathbf{b}$ shows the FWHM of the resonance at VL2 as a function of the parameter of $d$. c The geometrical parameters of the triangular patches inside the disk resonator. $\mathbf{d}$ The FSR between two resonances at VL1 and VL2 as a function of the $b$ parameter. e The magnitude of the electric field for the total system equipped with two metal patches inside the disk cavity for the resonance of the VL2 at $\lambda=$ $723.8 \mathrm{~nm}$. $\mathbf{f}, \mathbf{g}$ The magnitude of the electric field for the total system (without triangular patches) at $\lambda=680 \mathrm{~nm}$ (VL1) and $\lambda=694.8 \mathrm{~nm}$ (VL2), respectively. The fixed values of geometrical parameters of the newly added triangular metal patches in this subsection are as follows: $d=120 \mathrm{~nm}, b=100 \mathrm{~nm}$, and $h=80 \mathrm{~nm}$. The values of the other geometrical parameters are based on Table 1 .

Fig. 5a indicates the transmission spectra of the total system with two triangular metal patches incorporated inside the disk cavity (Fig. 5c) for different values of $b$ (base of the isosceles triangle). As it is depicted in Fig. 5d, the FSR between the two resonances at VL1 and VL2 can be adjusted by the variation of the base of triangular patches. All the parameters of the triangular patches are distinguished in Fig. 5c.

The inset of Fig. 5a shows the FWHMs of the VL1 and VL2 as a function of the $b$ parameter value. By increasing the $b$ parameter value (when $d=120 \mathrm{~nm}$ and $h=80 \mathrm{~nm}$ ), FSR between the peaks of VL1 and VL2 and the FWHMs of them will be increased (Fig. 5a). Therefore $b=100 \mathrm{~nm}$ is considered as an optimum value at which the FSR is 37.6 
$\mathrm{nm}$ between VL1 and VL2, and their FWHMs are $2.85 \mathrm{~nm}$ and $1.39 \mathrm{~nm}$, respectively. The quality factors of 240.7 and 520.7 are achieved for VL1 and VL2, respectively.

Fig. $5 \mathrm{~b}$ shows the transmission spectra of the total system, which is equipped with two triangular metal patches inside the disk cavity, as a function of the $d$ parameter (when $b=100 \mathrm{~nm}$ and $h=80 \mathrm{~nm}$ ) for different values of $d$. For the resonance of VL2, there is a trade-off between its FWHM (inset of Fig. 5b) and transmission intensity. $d=120 \mathrm{~nm}$ is chosen as an optimum value at which the transmission intensity of VL2 resonance is about 0.3 .

As it can be observed in Fig. 5a, the resonance at VL2 is more sensitive to variation of the $b$ parameter value with respect to the resonances at VL1 and NIR1. The increasing of the value of the $b$ parameter redshifts the VL2 more than the VL1 and NIR1. For finding the reason, the distribution of the electric fields inside the disk cavity is depicted in Figs. $5 \mathrm{f}$ and $\mathrm{g}$ for the two cases of VL1 and VL2 resonances, respectively. These two figures are for the total system without the triangular metal patches (i.e., $b=0$ ). It can be observed in Fig. $5 \mathrm{f}$ (for VL1) that the electric field exists mainly at the border of the disk cavity. Therefore, it does not engage with any metal patches inside the disk cavity. This is in contrast to the case of Fig. $5 \mathrm{~g}$ (for VL2), in which the electric field is not only located at the border of the disk cavity but also exists inside of it. This leads to the engagement of the electric field with any metal patches inside the disk cavity.

\subsection{Sensitivity and FOM of the proposed sensor}

In general, the transmission spectra of a MIM refractive index sensor depends on the materials and the geometrical parameters of the sensor. After fabrication, the material of metal and geometrical parameters of the structure are fixed. Thus, any change in the transmission spectra of the sensor will be related to the dielectric constant of the insulator material which is used in cavities. Fig. 6a shows the transmission spectra of the total system for six different dielectric constants. The dielectric constant of these cavities (The disk cavity, two pentagon cavities of the waveguide system, bottom rectangular cavity of the waveguide system, and the cavity that is located between two metals in the MIM waveguide as an insulator) has been increased from 1 to 1.05 , in steps of 0.01 and the transmission spectra has shifted consequently. Sensing performance is determined by the following parameters (Ameling et al. 2010):

$$
S=\frac{\Delta \lambda}{\Delta n}, F O M=\frac{S}{F W H M}
$$

The sensitivity (S) is the shift in the resonance wavelength as the result of changing the refractive index of the dielectric material inside the cavities. The FOM expresses the optical resolution of the sensor. According to the definition of the sensitivity, slopes of the curves in Fig. $6 \mathrm{~b}$ represent the sensitivity of corresponding resonance to the change of refractive index. Sensitivities of $666.2 \mathrm{~nm} / \mathrm{RIU}, 687.9 \mathrm{~nm} / \mathrm{RIU}, 888.9 \mathrm{~nm} / \mathrm{RIU}, 927.1 \mathrm{~nm} / \mathrm{RIU}$, and $1012.6 \mathrm{~nm} / \mathrm{RIU}$ are achieved for the VL1, VL2, NIR1, NIR2, and NIR3 resonances, respectively. Also, the FWHMs of $2.67 \mathrm{~nm}, 1.15$ $\mathrm{nm}$, and $5.65 \mathrm{~nm}$ are achieved (when $n=1$ ) for the VL1, VL2, and NIR1 resonances, respectively. These lead to FOM of $249.5 \mathrm{RIU}^{-1}, 598.1 \mathrm{RIU}^{-1}$ and $157.3 \mathrm{RIU}^{-1}$ for the mentioned resonances, respectively. 

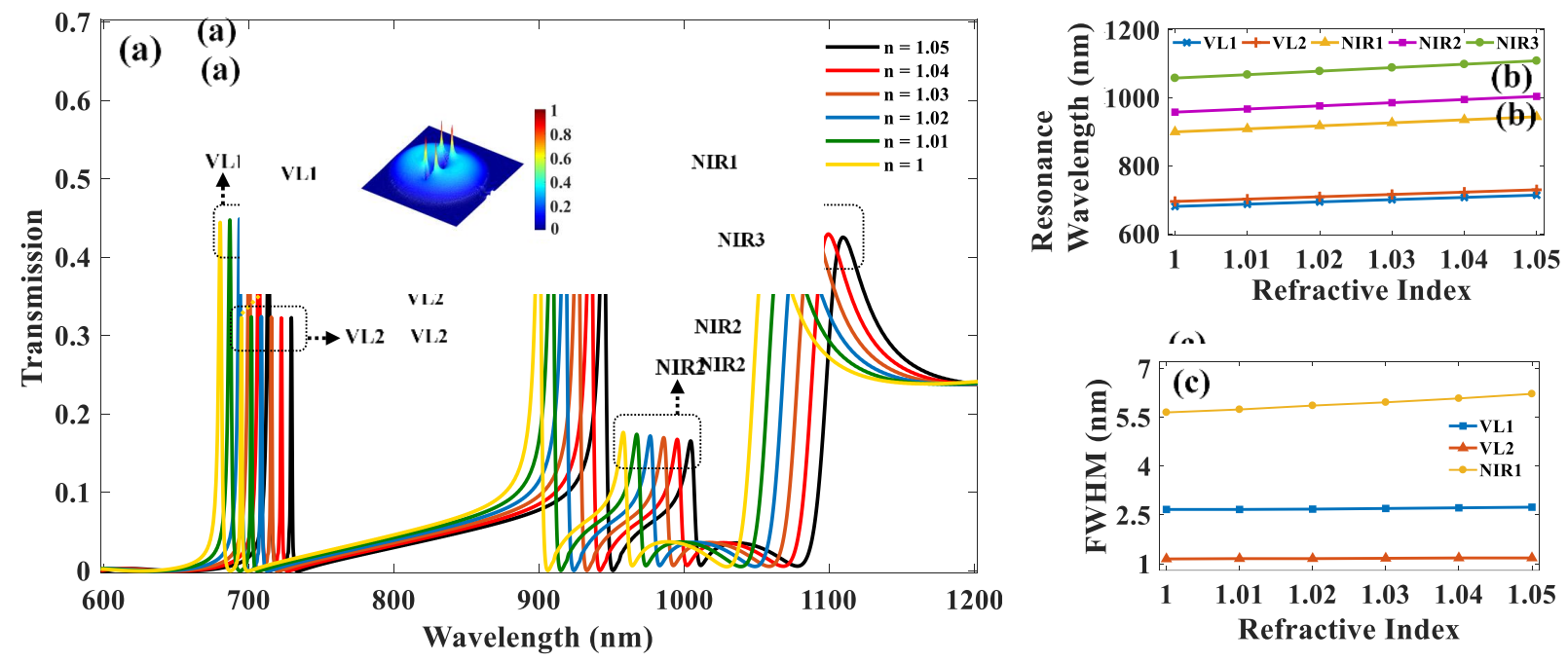

Fig. 6 Based on Table 1 values of the parameters: a The transmission spectra of the total system for six distinct values of the refractive index of the cavities. In a, the inset shows the electric field distribution inside the disk cavity at $\lambda=694.8 \mathrm{~nm}$. $\mathbf{b}$ The peak wavelengths and $\mathbf{c}$ the FWHMs of the resonances as function of the refractive index of the cavities.

The achieved FOMs, especially for the case of VL2 $\left(598.1 \mathrm{RIU}^{-1}\right)$, are much higher than the other works which are reported recently (as shown in Table 2). It is essential to be mentioned that there are some works in the literature reporting values of up to several thousands for the FOM of their sensors. But the FOM definition of those works is different (Kazanskiy et al. 2020).

Table 2 Comparison of Sensitivity (S) and figure of merit (FOM) of the proposed sensor (for the total system) with other recent works.

\begin{tabular}{cccc}
\hline Reference & $\mathrm{S}(\mathrm{nm} / \mathrm{RIU})$ & $\left.\mathrm{FOM}_{(\mathrm{RIU}}{ }^{-1}\right)$ & Operating Region \\
\hline (Butt et al. 2021) & 1320 & 16.7 & Near Infra Red \\
(Wang et al. 2019) & 1114.3 & 55.71 & Near Infra Red \\
(Zafar, Salim 2015) & 1060 & 176.7 & Near Infra Red \\
(Zhang et al. 2018) & 1060 & 203.8 & Near Infra Red \\
(Danaie, Shahzadi 2019) & 636 & 211.3 & Near Infra Red \\
(Zhang et al. 2020) & 579 & 378 & Visible Light \\
This study & 687.9 & 598.1 & Visible Light \\
\hline
\end{tabular}

As shown in subsection 3.2, the Fano resonances arise by the interaction of the broad spectral line induced by the waveguide system with narrow discrete resonances induced by the disk cavity. The transmission spectra of the waveguide system has its own part in the formation of the Fano resonances (Fig. 3b), and as it was shown in subsection 3.1 , it is highly function of geometrical parameters of the waveguide system. The proposed waveguide system in this work has seven different geometrical parameters (Fig. 1b), which for brevity, the results of optimization for only two of them $\left(x\right.$ and $\left.g_{2}\right)$ was mentioned in subsection 3.3. unlike the simple MIM waveguide (which are applied in (Zhang et al. 2018; Zhang et al. 2020), with no additional cavity, that has only the geometrical parameter of width (width of insulator between two metal) to be evaluated, in this work, more decisive geometrical parameters on transmission spectra of the total system provided more variables to be applied in the optimization of the FOM of the resonances and resulted in FOM of 598.1 $\mathrm{RIU}^{-1}$ for the resonance of VL2.

The sensitivities reported in some of these works are considerably higher than the mentioned sensitivity of the proposed sensor at this work, but their achievements are only for one resonance which is located in the near infra red 
region. By comparing their reported sensitivities for other resonances, it can be concluded that sensitivities which are located in the visible light region are lower than those which are located in the near infra red region. (Zhang et al. 2016), (Zhang et al. 2020), (Wang et al. 2019) and (Zhang et al. 2018) have reported sensitivities of $596 \mathrm{~nm} / \mathrm{RIU}, 579$ $\mathrm{nm} / \mathrm{RIU}, 623.8 \mathrm{~nm} / \mathrm{RIU}$, and $708 \mathrm{~nm} / \mathrm{RIU}$, respectively for their resonances in the visible light region.
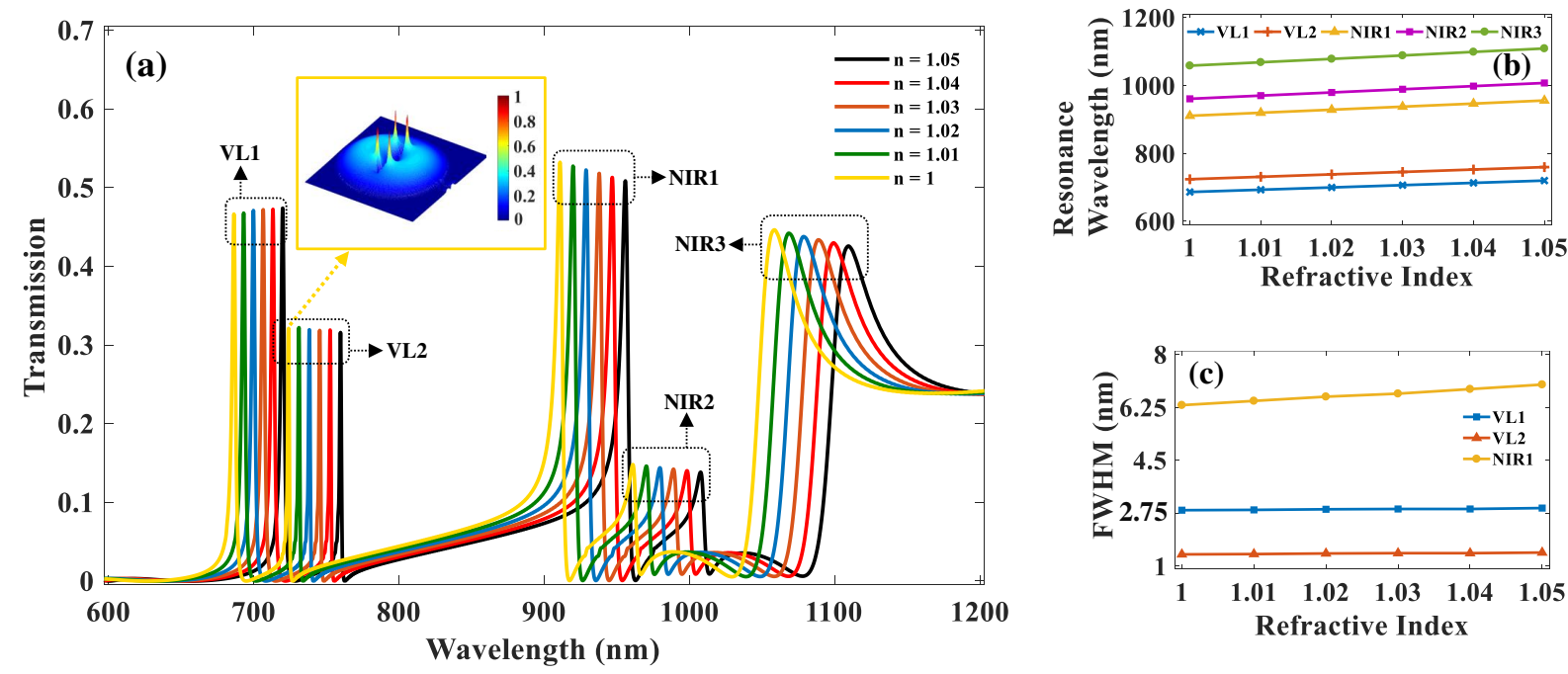

Fig. 7 a The transmission spectra of the total system equipped with two triangular silver patches inside the disk cavity, for six distinct refractive index values. In $\mathbf{a}$, the inset shows the electric field distribution inside the disk cavity for $\lambda=723.8 \mathrm{~nm}$. $\mathbf{b}$ The peak wavelengths and $\mathbf{c}$ the FWHMs of the resonances as function of the refractive index of the cavities. For this figure, all the fixed values of the geometrical parameters are the same as the values mentioned in the caption of Fig. 5.

In order to extract the sensor parameters ( $\mathrm{S}$ and $\mathrm{FOM}$ ) for the total system equipped with two triangular silver patches inside the disk cavity (Fig. 5c), the same procedures that applied to the total system itself, have been repeated. Fig. 7a shows the transmission spectra of the total system equipped with two triangular silver patches inside the disk cavity, for six different refractive index values which are applied to the material of the cavities. The refractive index of the dielectric material inside the cavities is increased from 1 to 1.05 in steps of 0.01 . According to Fig. 7b, sensitivity (or $\Delta \lambda / \Delta \mathrm{n}$ ) of $672.5 \mathrm{~nm} / \mathrm{RIU}, 715.6 \mathrm{~nm} / \mathrm{RIU}, 901.1 \mathrm{~nm} / \mathrm{RIU}, 933.4 \mathrm{~nm} / \mathrm{RIU}$, and $1017.2 \mathrm{~nm} / \mathrm{RIU}$ are achieved for the resonances located at VL1, VL2, NIR1, NIR2, and NIR3, respectively. Also, FWHMs of $2.85 \mathrm{~nm}, 1.39 \mathrm{~nm}, 6.32 \mathrm{~nm}$ are achieved (when $n=1$ ) for the VL1, VL2, and NIR1 resonances, respectively. These lead to FOM of $235.9 \mathrm{RIU}^{-1}$, $514.8 \mathrm{RIU}^{-1}$ and $142.5 \mathrm{RIU}^{-1}$ for resonances of VL1, VL2, and NIR1, respectively. By comparing the sensitivities for two cases of the total system with or without triangular silver patches inside the disk cavity, it can be concluded that sensitivities of the all resonances are increased in the case that metal patches are applied inside the disk resonator. However, the sensitivity of VL2 is increased remarkably with respect to other resonances. This is because the electric field in the case of VL2 is engaged with the triangular patches inside the disk cavity. It can be observed in the inset of Fig. 7a that the maximum value of the electric field magnitude has been increased in distinct spots (at two base corners of the silver triangles) inside the disk cavity from $4.8 \mathrm{~V} / \mathrm{m}$ (without metal patches inside the disk resonator as it is depicted in the inset of Fig. 6a) to $14.4 \mathrm{~V} / \mathrm{m}$ (with metal patches inside the disk resonator). Therefore, the presence of the silver triangular patches inside the disk cavity enhances the mechanism of sensing for the resonance of VL2. Moreover, the sensitivity of resonance of NIR3 has experienced very little increase which is because this resonance is induced by the waveguide system itself, and the presence of silver triangular patches has no impact on it.

\subsection{Evaluating the stability of Fano resonances of proposed structure}

The goal of this subsection is to explore deviations of the transmission spectra of the total system due to defects in delicate sections of its structure. These defects may appear in the fabrication process. 

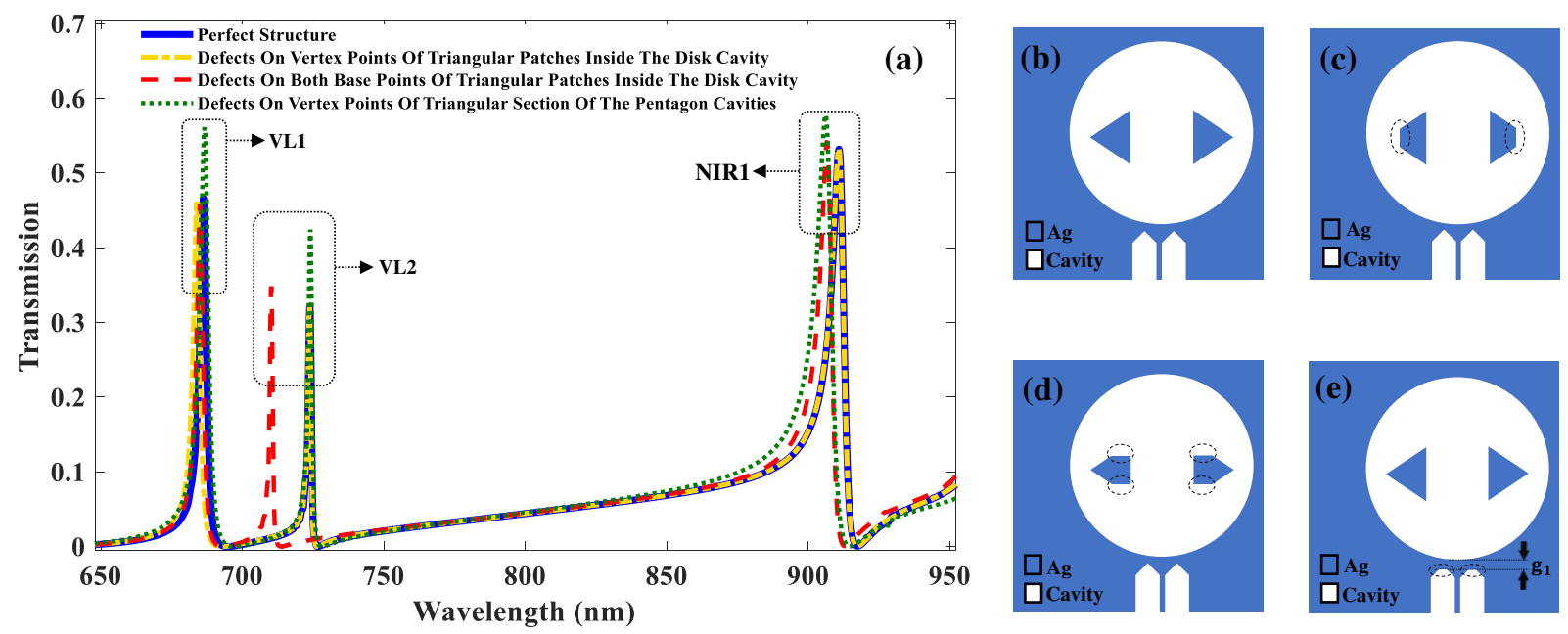

Fig. 8 a The transmission spectra of the total system with two triangular silver patches inside the disk cavity for the cases of $\mathbf{b}$ the perfect structure, $\mathbf{c}$ the structure with defects at vertex points of the two silver patches, $\mathbf{d}$ the structure with defects at both base points of the two silver patches, and $\mathbf{e}$ the structure with defects at vertex points of the triangular part of the two pentagon cavities. In $\mathbf{e}$, the parameter of $g_{1}$ is redefined. For this figure, all the fixed values of the geometrical parameters are the same as the values mentioned in the caption of Fig. 5. The area of the defects that are highlighted in $\mathbf{c}, \mathbf{d}$, and $\mathbf{e}$ inside the black-dashed ovals are $25 \mathrm{~nm} \times 20 \mathrm{~nm}, 20 \mathrm{~nm} \times 32 \mathrm{~nm}$, and $20 \mathrm{~nm} \times 10 \mathrm{~nm}$, respectively.

Fig. 8a compares the transmission spectra of the total system with two triangular silver patches inside the disk cavity when the structure is perfect and when there are defects in delicate parts of the structure. Fig. 8b shows the perfect structure layout, Fig. 8c shows triangular defects with a base of $25 \mathrm{~nm}$ and height of $20 \mathrm{~nm}$ at vertex points of the two triangular silver patches, Fig. 8d shows triangular defects with a base of $20 \mathrm{~nm}$ and height of $32 \mathrm{~nm}$ at both base points of the two triangular silver patches, and Fig. 8e shows triangular defects with a base of $20 \mathrm{~nm}$ and height of $10 \mathrm{~nm}$ at vertex points of the triangular parts of the two pentagon cavities. In Fig. 8e, the gap between the waveguide system and the disk cavity or $g_{1}$ is redefined due to defects at the top of the waveguide system. For the resonance of VL2, FWHMs of $1.39 \mathrm{~nm}, 1.38 \mathrm{~nm}, 1.28 \mathrm{~nm}$, and $1.67 \mathrm{~nm}$ are achieved in: the perfect structure (Fig. 8b), the structure with defects on vertex points of the silver patches (Fig. 8c), the structure with defects on both base points of silver patches (Fig. 8d) and the structure with defects on vertex points of the triangular part of the pentagon cavities (Fig. 8e), respectively. Even though these defects have considerable sizes, but remarkable changes for the transmission spectra of the sensor have not happened. In Fig. 8d, the resonance of VL2 has blueshifted, which is compatible with the results achieved in subsection 3.4 (the wavelength resonance of VL2 can be tuned by the parameter of the base of the triangular metal patches (or $b$ ) inside the disk cavity). The relative stability of the transmission spectra of the sensor, regarding the presence of the large area of defects, verifies its dependability. The reliability of the transmission spectra of the proposed sensor at remarkable ranges of the geometrical parameters (concluded in subsection 3.3), and also at the presence of the defects makes this structure a suitable choice for fabrication.

\section{Conclusion}

In summary, we have proposed a nano-scaled plasmonic refractive index sensor whose Fano resonances arise from interference of SPP modes between a disk cavity and a waveguide system. The waveguide system consists of a MIM waveguide attached to three polygonal cavities. By adjusting the geometrical parameters of the proposed structure, resonance wavelengths and FWHMs of its resonances have been set. For the desired resonance, the sensitivity of the $687.9 \mathrm{~nm} / \mathrm{RIU}$ with FWHM of the $1.15 \mathrm{~nm}$, quality factor of the 604.1 , and FOM of the $598.1 \mathrm{RIU}^{-1}$ has been achieved in the visible light region. By incorporating silver patches inside the disk cavity, the tunability of the FSR between resonances and also enhancement of sensing mechanism has been discussed. Ultimately, the stability of the proposed sensor has been evaluated by extracting its transmission spectra in the cases with the most likely defects in the fabrication process. All the results were obtained by 2D-FDTD simulations. This structure with such high optical resolution could be used in highly integrated photonic circuits. 
Conflict of interest All the authors declare that none of us have no conflict of interests.

\section{References}

Akhavan, A., Ghafoorifard, H., Abdolhosseini, S., Habibiyan, H.: Metal-insulator-metal waveguide-coupled asymmetric resonators for sensing and slow light applications. IET Optoelectron. 12, 220-227 (2018)

Ameling, R., Langguth, L., Hentschel, M., Mesch, M., Braun, P. V., Giessen, H.: Cavity-enhanced localized plasmon resonance sensing. Appl. Phys. Lett. 97, 253116 (2010)

Barnes, W. L., Dereux, A., Ebbesen, T. W.: Surface plasmon subwavelength optics. Nature 424, 824-830 (2003)

Butt, M., Khonina, S., Kazanskiy, N.: Plasmonic refractive index sensor based on MIM square ring resonator, in: 2018 International Conference on Computing, Electronic and Electrical Engineering (ICE Cube), pp. 1-4 (2018)

Butt, M., Khonina, S., Kazanskiy, N.: Plasmonic refractive index sensor based on metal-insulator-metal waveguides with high sensitivity. J. Mod. Opt. 66, 1038-1043 (2019)

Butt, M., Khonina, S., Kazanskiy, N.: Metal-insulator-metal nano square ring resonator for gas sensing applications. Waves Random Complex Media 31, 146-156 (2021)

Chen, Y., Xu, Y., Cao, J.: Fano resonance sensing characteristics of MIM waveguide coupled square convex ring resonator with metallic baffle. Results Phys. 14, 102420 (2019)

Chen, Z., Yu, L.: Multiple Fano resonances based on different waveguide modes in a symmetry breaking plasmonic system. IEEE Photonics J. 6, 1-8 (2014)

Danaie, M., Shahzadi, A.: Design of a high-resolution metal-insulator-metal plasmonic refractive index sensor based on a ringshaped Si resonator. Plasmonics 14, 1453-1465 (2019)

Deng, Y., Cao, G., Yang, H.: Tunable Fano resonance and high-sensitivity sensor with high figure of merit in plasmonic coupled cavities. Photonics Nanostructures - Fundam. Appl. 28, 45-51 (2018)

Elsayed, M. Y., Ismail, Y., Swillam, M. A.: Semiconductor plasmonic gas sensor using on-chip infrared spectroscopy. Appl. Phys. A 123, 113 (2017)

Fang, Y., Wen, K., Li, Z., Wu, B., Chen, L., Zhou, J., Zhou, D.: Multiple Fano resonances based on end-coupled semi-ring rectangular resonator. IEEE Photonics J. 11, 1-8 (2019)

Gierak, J., Madouri, A., Biance, A., Bourhis, E., Patriarche, G., Ulysse, C., Lucot, D., Lafosse, X., Auvray, L., Bruchhaus, L.: Sub-5 nm FIB direct patterning of nanodevices. Microelectron. Eng. 84, 779-783 (2007)

Hajshahvaladi, L., Kaatuzian, H., Danaie, M.: Design and analysis of a plasmonic demultiplexer based on band-stop filters using double-nanodisk-shaped resonators. Opt. Quantum Electron 51, 1-16 (2019)

He, M.-D., Liu, J.-Q., Gong, Z.-Q., Luo, Y.-F., Chen, X., Lu, W.: Plasmonic splitter based on the metal-insulator-metal waveguide with periodic grooves. Opt. Commun. 283, 1784-1787 (2010)

Johnson, P. B., Christy, R.-W.: Optical constants of the noble metals. Phys. Rev. B Condens. Matter 6, 4370 (1972)

Kaatuzian, H., Taheri, A. N., Applications of Nano-Scale Plasmonic Structures in Design of Stub Filters-A Step Towards Realization of Plasmonic Switches: BoD-Books on Demand, p. 93 (2015)

Karimi, Y., Kaatuzian, H., Tooghi, A., Danaie, M.: All-Optical Plasmonic Switches Based on Fano Resonance in an X-Shaped Resonator Coupled to Parallel Stubs for Telecommunication Applications. Optik 243, 167424 (2021)

Kazanskiy, N., Khonina, S., Butt, M.: Plasmonic sensors based on Metal-insulator-metal waveguides for refractive index sensing applications: A brief review. Physica E Low Dimens. Syst. Nanostruct. 117, 113798 (2020)

Khani, S., Danaie, M., Rezaei, P.: Plasmonic all-optical metal-insulator-metal switches based on silver nano-rods, comprehensive theoretical analysis and design guidelines. J. Comput. Electron. 20, $442-457$ (2021)

Lu, H., Liu, X., Mao, D., Wang, G.: Plasmonic nanosensor based on Fano resonance in waveguide-coupled resonators. Optics Letters 37, 3780-3782 (2012)

Lu, H., Wang, G., Liu, X.: Manipulation of light in MIM plasmonic waveguide systems. Sci. Bull. 58, 3607-3616 (2013)

Luk'yanchuk, B., Zheludev, N. I., Maier, S. A., Halas, N. J., Nordlander, P., Giessen, H., Chong, C. T.: The Fano resonance in plasmonic nanostructures and metamaterials. Nat. Mater. 9, 707-715 (2010)

Maier, S. A., Plasmonics: fundamentals and applications. Springer Science \& Business Media, (2007)

Moeinimaleki, B., Kaatuzian, H., Livani, A. M.: Design and Simulation of Nano Scale Plasmonic Temperature Sensor using Ethanol-Filled Rectangular Cavity Equipped with Polygonal Patches, in: Asia Communications and Photonics Conference, p. T4A. 267 (2021)

Ou, X., Yang, Y., Sun, F., Zhang, P., Tang, B., Li, B., Liu, R., Liu, D., Li, Z.: Wide-range, ultra-compact, and high-sensitivity ring resonator biochemical sensor with CMOS-compatible hybrid plasmonic waveguide. Opt. Express 29, 1905819067 (2021)

Ouyang, Q., Zeng, S., Jiang, L., Qu, J., Dinh, X.-Q., Qian, J., He, S., Coquet, P., Yong, K.-T.: Two-dimensional transition metal dichalcogenide enhanced phase-sensitive plasmonic biosensors: theoretical insight. J. Phys. Chem. 121, 6282-6289 (2017)

Ozbay, E.: Plasmonics: merging photonics and electronics at nanoscale dimensions. Science 311, 189-193 (2006) 
Setareh, M., Kaatuzian, H.: Sensitivity enhancement of a surface plasmon resonance sensor using Blue Phosphorene/MoS2 hetero-structure and barium titanate. Superlattices Microstruct. 153 (2021)

Shafagh, S. G., Kaatuzian, H., Danaie, M.: Analysis, design and simulation of MIM plasmonic filters with different geometries for technical parameters improvement. Commun. Theor. Phys. 72, 085502 (2020)

Wang, G., Lu, H., Liu, X., Gong, Y.: Numerical investigation of an all-optical switch in a graded nonlinear plasmonic grating. Nanotechnology 23, 444009 (2012)

Wang, M., Zhang, M., Wang, Y., Zhao, R., Yan, S.: Fano resonance in an asymmetric MIM waveguide structure and its application in a refractive index nanosensor. Sensors 19, 791 (2019)

Wen, K., Yan, L., Pan, W., Luo, B., Guo, Z., Guo, Y.: Wavelength demultiplexing structure based on a plasmonic metalinsulator-metal waveguide. J. Opt. 14, 075001 (2012)

Wu, T., Liu, Y., Yu, Z., Ye, H., Peng, Y., Shu, C., Yang, C., Zhang, W., He, H.: A nanometeric temperature sensor based on plasmonic waveguide with an ethanol-sealed rectangular cavity. Opt. Commun. 339, 1-6 (2015)

Wu, W., Yang, J., Zhang, J., Huang, J., Chen, D., Wang, H.: Ultra-high resolution filter and optical field modulator based on a surface plasmon polariton. Opt. Lett. 41, 2310-2313 (2016)

Yan, S., Zhang, M., Zhao, X., Zhang, Y., Wang, J., Jin, W.: Refractive index sensor based on a metal-insulator-metal waveguide coupled with a symmetric structure. Sensors 17, 2879 (2017)

Yu, S., Zhao, T., Yu, J., Pan, D.: Tuning multiple Fano resonances for on-chip sensors in a plasmonic system. Sensors 19, 1559 (2019)

Yu, Y., Si, J., Ning, Y., Sun, M., Deng, X.: Plasmonic wavelength splitter based on a metal-insulator-metal waveguide with a graded grating coupler. Opt. Lett. 42, 187-190 (2017)

Zafar, R., Salim, M.: Enhanced figure of merit in Fano resonance-based plasmonic refractive index sensor. IEEE Sens. J. 15, 6313-6317 (2015)

Zhan, G., Liang, R., Liang, H., Luo, J., Zhao, R.: Asymmetric band-pass plasmonic nanodisk filter with mode inhibition and spectrally splitting capabilities. Opt. Express 22, 9912-9919 (2014)

Zhang, Z., Luo, L., Xue, C., Zhang, W., Yan, S.: Fano resonance based on metal-insulator-metal waveguide-coupled double rectangular cavities for plasmonic nanosensors. Sensors 16, 642 (2016)

Zhang, Z., Yang, J., Han, Y., He, X., Huang, J., Chen, D.: Hybridization-induced resonances with high-quality factor in a plasmonic chipscale ring-disk nanocavity. Waves Random Complex Media, 1-10 (2020)

Zhang, Z., Yang, J., He, X., Zhang, J., Huang, J., Chen, D., Han, Y.: Plasmonic refractive index sensor with high figure of merit based on concentric-rings resonator. Sensors 18, $116(2018)$ 